\title{
Cost Savings and Efficacy in Management of Paracetamol Poisoning in a 23-hours Emergency Department Observation Unit: A Comparison to Inpatient Care
}

Kelvin K. Kuan ${ }^{1}$, Hoon Chin Lim ${ }^{1}$, Geraldine Goh ${ }^{2}$, Gabriel S. Arciaga ${ }^{1}$, Pak Liang Goh ${ }^{1}$, Rupeng Mong ${ }^{1}$ , Wai Leng Chow ${ }^{2}$, Hock Heng Tan ${ }^{1}$

1. Accident and Emergency, Changi General Hospital / Singhealth, Singapore, SGP 2. Health Sciences Research, Changi General Hospital / Singhealth, Singapore, SGP

Corresponding author: Kelvin K. Kuan, kelvin.kuan.k.b@singhealth.com.sg

\section{Abstract}

\section{Introduction}

Emergency department observation units (EDOU) have been shown to be effective in decreasing hospitalization rates and length of stay (LOS) for various conditions. However, cost savings and efficacy in the management of poisoning in EDOU have not been widely studied. The objective of our study is to compare the costs and effectiveness of managing paracetamol poisoned patients in the EDOU with those treated in the inpatient wards.

\section{Methods}

We conducted a historical controlled observational study comparing paracetamol-poisoned patients (who received at least 21 hours of IV N-acetylcysteine [NAC]) admitted to the EDOU during 2013-2014 with similar patients admitted to inpatient ward during 2011, 2013-2014.

\section{Results}

We found 136 patients admitted to the inpatient ward and 95 to our EDOU due to paracetamol poisoning but only 78 and 39 patients respectively fulfilled the inclusion criteria. Between the EDOU and inpatient ward groups, we found similar demographics, poisoning presentation, treatment, and adverse event profiles. There were no fatalities and only two patients (one from each group) developed hepatotoxicity. The "medical" length of stay was 31.9 hours shorter in the EDOU group compared to the inpatient ward group (23.3 versus 55.2 hours). EDOU patients have statistically significant savings (comparing bill size) of $S \$ 784$ per patient.

Received 06/24/2019 Review began $06 / 30 / 2019$ Review ended 11/30/2019 Published 12/05/2019

๑) Copyright 2019

Kuan et al. This is an open access article distributed under the terms of the Creative Commons Attribution License CC-BY 3.0., which permits unrestricted use, distribution, and reproduction in any medium, provided the original author and source are credited.

\section{Conclusions}

Admission to the EDOU resulted in significant cost savings and 58\% decreased LOS when compared to inpatient wards. The EDOU is a cost-effective and safe alternative for the management of selected paracetamol poisonings requiring NAC. Further studies would be needed to verify these results.

Categories: Emergency Medicine, Internal Medicine, Epidemiology/Public Health

Keywords: acetaminophen, paracetamol, poisoning, length of stay, cost-savings, n-acetylcysteine, hepatotoxicity, observation ward

\section{Introduction}

Paracetamol poisoning is the most common pharmaceutical poisoning in developed countries including Singapore [1-5]. N-acetylcysteine (NAC) is an effective antidote. A standard intravenous (IV) dose of NAC $(300 \mathrm{mg} / \mathrm{kg}$ ) is given over 21 hours, usually requiring admission to the hospital inpatient ward. In one paper, the admission rate from the Emergency Department (ED) for paracetamol poisoning was approximately $45 \%$ for an average duration of 3.1 days [2]. Locally, admission duration was comparable at three days [6, 7].

Since its inception, observation units have been shown to be effective for the management of mild to moderate poisoning [8]. Protocol-driven emergency department observation units (EDOU) generally have a limited observation period of 24 hours, presenting a challenge to the administration of NAC [9]. However, studies have shown that management of paracetamol poisonings in an EDOU by a multidisciplinary protocol can help cut down hospital length of stay (LOS) [7, 10]. Although it is acknowledged that EDOU helps to decrease LOS and cost, there are no comparative studies on cost savings from the management of poisoning cases in EDOU versus inpatient admission [7, 9].

Our hospital EDOU protocol for poisoning commenced in end-2011 [11]. Thereafter, paracetamol-poisoned cases requiring NAC may be managed in the EDOU or the inpatient wards. We conducted a study to compare the effectiveness, safety, and costs of managing these patients who were admitted to the EDOU with those admitted to the inpatient wards. 


\section{Materials And Methods \\ Study setting and population}

Changi General Hospital is one of six public tertiary teaching hospitals in Singapore, with an annual estimated emergency department (ED) attendance of 150,000, representing about 15\% of all ED attendances amongst the nation's public hospitals [12].

NAC is started for paracetamol-poisoned patients with the possible risk of hepatotoxicity based on standard recommendations for both acute and repeated supratherapeutic ingestions (RSTI) [13]. Occasionally, empirically started NAC is stopped when subsequent assessment found it unnecessary.

Our EDOU poisoning protocols admits patients 12 years or older, who have features of poisoning that require treatment or extended observation and are deemed likely to improve within 24 hours based on the type of poisoning and predicted clinical course [11]. Patients who do not fulfill EDOU criteria, or present with unstable vital signs, any evidence of end-organ injury or concurrent medical issues deemed to require inpatient management are excluded. Our EDOU had nine beds, managed by an emergency physician (EP) and the team in rotating shifts with support from the toxicology service.

EPs decide on the disposition of patients presenting with paracetamol poisoning. A proportion of patients who fulfill the admission criteria to EDOU may get admitted to the inpatient ward instead. The reasons may include EP's unfamiliarity with the EDOU protocol, lack of EDOU beds and patient preference.

Management in the EDOU aims to complete clinical care and formulate a psychosocial care plan within 23 hours. Standard NAC therapy is given as required for paracetamol poisoning. Patients may be transferred to the inpatient medical ward if they require extended NAC therapy or further evaluation of other medical conditions. Following an initial assessment by psychiatry doctors in the EDOU, patients may be discharged with an outpatient psychiatry appointment or transferred to an inpatient psychiatric service. This inpatient psychiatric service could be at our hospital (hospital psychiatric facility) or at a nearby mental health institution (external psychiatric facility). Patients admitted to the inpatient medical wards are treated for their poisoning as above and undergo similar psychiatric evaluation and disposition as needed.

\section{Study design and analysis}

This is a historical controlled observational study of paracetamol poisoned patients presenting to our ED who were treated with at least a standard course of NAC either in the inpatient medical ward or EDOU.

Case Selection

All patients admitted to the hospital under the International Classification of Diseases, Ninth Revision, Clinical Modification (ICD-9-CM) code of 965 (poisoning by analgesics, antipyretics and antirheumatics) were obtained from computer records from January to December of 2011, 2013 and 2014. EDOU cases admitted under poisoning protocols in 2013 and 2014 were reviewed. Cases that did not involve paracetamol poisoning were excluded. We did not include 2012 as the EDOU had just started and the number of cases admitted there was low.

Cases selected for comparison: 1. received at least a standard 300mg/kg dose of IV NAC given over 21 hours for paracetamol poisoning and 2. met the criteria for EDOU admission. All inpatient ward admissions were reviewed by two study authors for consensus for the latter criteria. This allowed us to compare paracetamol poisoning cases of similar severity by excluding patients who are too ill for EDOU and patients who did not require NAC.

Patients with mildly elevated initial aminotransferase (AT) [either alanine aminotransferase (ALT) or aspartate aminotransferase (AST)] levels less than two times the upper limit of normal (ULN) were included in our study. This reflects the real-world practice at our institution, where we admit such patients to the EDOU. The mildly elevated AT may be due to conditions other than paracetamol poisoning [14, 15]. The transaminases of these patients were trended during the EDOU stay. Acute liver injury is defined as AT $>150$ $\mathrm{IU} / \mathrm{L}$ and hepatotoxicity is defined as AT $>1000 \mathrm{IU} / \mathrm{L}[16]$.

Group Comparison

Patients admitted to the EDOU in 2013 and 2014 were compared to those admitted to inpatient wards in 2011, 2013 and 2014. A comparative analysis was performed on patient demographics, outcome, adverse events, and hospital resource use, namely LOS and estimated costs.

We grouped selected cases into four groups for comparison: 1. pre-EDOU inpatient ward group (inpatient admissions in 2011); 2. post-EDOU inpatient ward group (inpatient admissions in 2013 and 2014); 3. all inpatient ward group (the combination of both groups 1 and 2) termed as a "ward" group; 4 . EDOU group. Statistical comparisons were made between groups 2 and 4 and between groups 3 and 4 .

"Medical" LOS by hours is defined as the length of hospital stay from the time of admission to the time of hospital discharge, excluding time spent in any inpatient psychiatric facility. This does not include the time 
spent in the ED. This allowed us to compare the LOS required solely for the medical management of the patients. Patients in the EDOU group who were transferred to an inpatient medical ward for further management were analyzed with the EDOU group.

All data was entered directly into a standardized Microsoft Excel (Microsoft, Redmond,

Washington, US) spreadsheet. A descriptive analysis was performed. Categorical data are presented as percentage frequencies, while continuous data are presented as medians with inter-quartile ranges (IQR). Statistical analyses were performed with data analytic software on GraphPad (GSL Biotech LLC, Chicago, US). The level for statistical significance was set at $p<0.05$.

\section{Financial Analysis}

To calculate the financial cost of the hospital visit, we could not exclude the LOS when the patient was transferred under our hospital psychiatric facility. This is because the extraction of cost is based on the whole LOS duration in our hospital and hence, we reported it as "actual LOS" for the whole hospital stay, with the costs involved. This cost only includes expenses within the hospital stay and ends when patients were discharged or transferred out of the hospital. All costs are presented in Singapore dollars (S\$).

The primary cost outcome was the bill size difference between the two cohorts. To compare across the years, the quantity for each service code was inflation-adjusted using a price list generated in March 2018 for each patient. A generalized linear model using the gamma family and log link was used to predict costs with cohort and LOS as independent variables. The choice of the gamma distribution was confirmed by the modified Park test. Several goodness-of-fit tests (Pearson correlation, Pregibon's link and the modified Hosmer-Lemeshow tests had insignificant p-values) indicated the acceptability of the log-link function. Using recycled predictions, the cost difference between the cohorts was computed assuming the same covariate balance in both groups. As the cost and LOS data was skewed, bootstrapped $95 \%$ confidence intervals (CIs) around the means are reported.

\section{Results}

Of the 153 ward admissions (91 cases in 2011, 36 in 2013 and 26 in 2014) identified under ICD-9-CM code 965 in the study period, 136 involved paracetamol poisoning. We found 220 EDOU admissions (66 cases in 2013 and 154 cases in 2014) under the poisoning protocol, of which 95 involved paracetamol poisoning. We used 78 cases ("ward" group) and 39 cases (EDOU group) for comparison. We excluded 58 patients from the "ward" group and 56 patients from the EDOU group. The reasons for their exclusion are found in Figure 1 below.

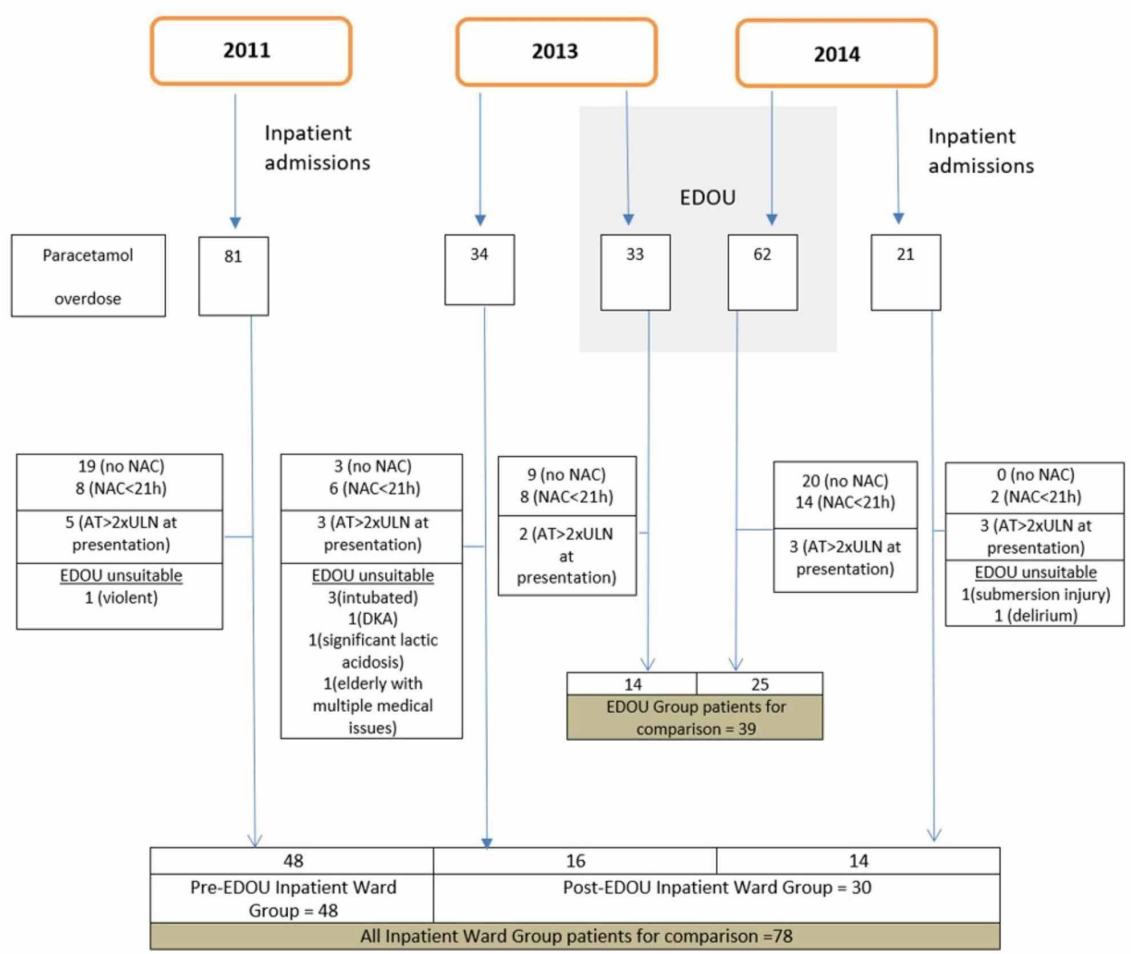

FIGURE 1: Patient inclusion and exclusion

NAC - N-acetylcysteine; AT - aminotransferase; ULN - upper limit of normal; EDOU - emergency department observation units 
Table 1 describes the demographics and characteristics of these patients. Comparison of the different groups ( 2 versus 4 and 3 versus 4 ) showed that they were similar in age and distribution, with no statistical differences in gender, race and presenting symptoms.

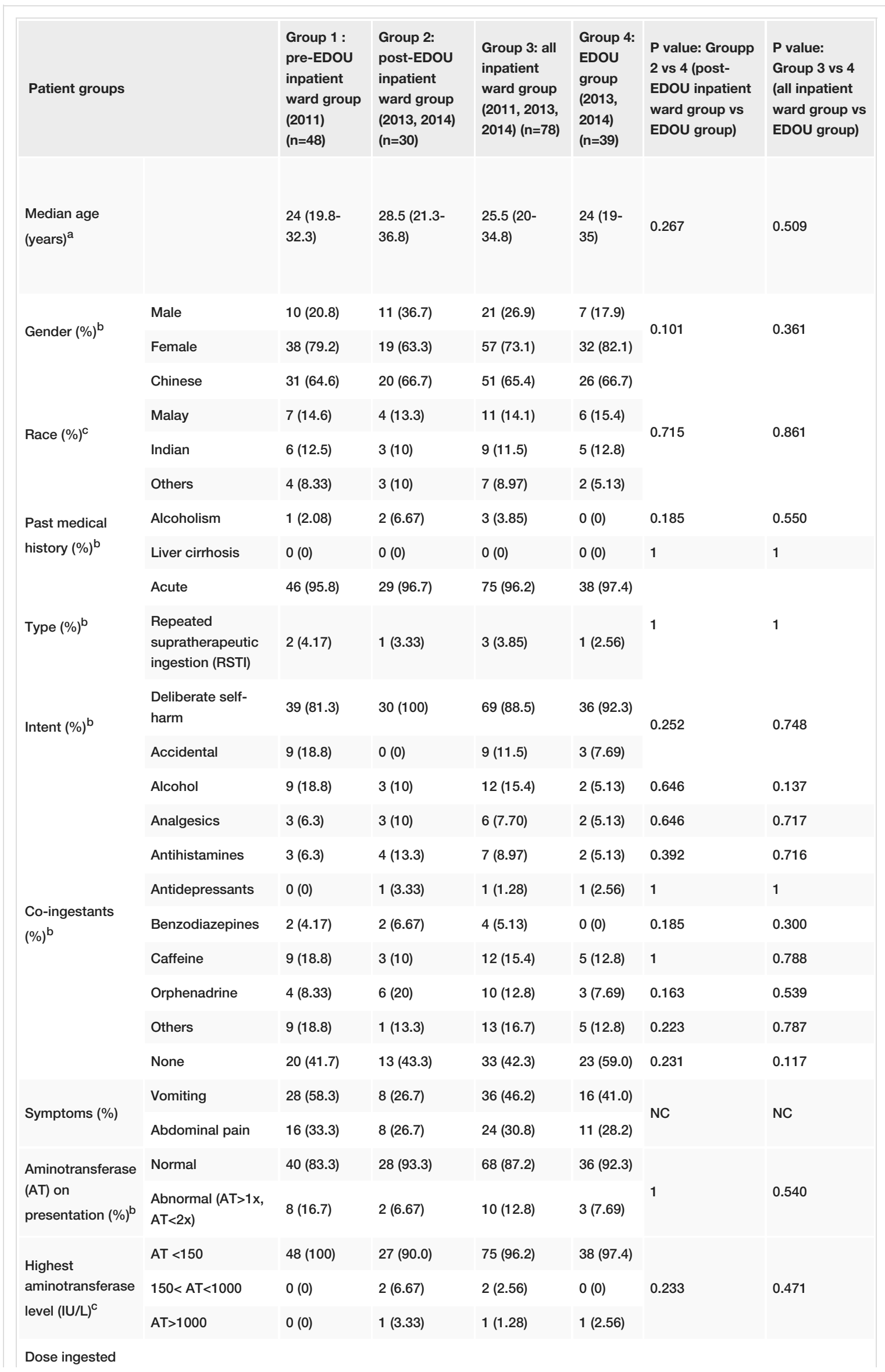




\section{Cureus}

\begin{tabular}{|c|c|c|c|c|c|c|c|}
\hline $\begin{array}{l}\text { (g) median } \\
\text { (interquartile } \\
\text { range) }\end{array}$ & & $10(9-16.0)$ & $11(9-18)$ & $10(9-17.3)$ & $\begin{array}{l}12(10- \\
15)\end{array}$ & 0.902 & 0.641 \\
\hline Decontamination & None & $37(77.1)$ & $27(90)$ & $64(82.1)$ & $32(82.1)$ & \multirow{2}{*}{0.496} & \multirow{2}{*}{1} \\
\hline$(\%)^{b}$ & Activated charcoal & $11(22.9)$ & $3(10)$ & 14 (17.9) & 7 (17.9) & & \\
\hline \multirow{2}{*}{$\begin{array}{l}\text { NAC duration } \\
(\%)^{b}\end{array}$} & 20-21 hours & $43(89.6)$ & $27(90)$ & 70 (89.7) & $36(92.3)$ & \multirow{2}{*}{1} & \multirow{2}{*}{0.750} \\
\hline & $>21$ hours & $5(10.4)$ & $3(10)$ & $8(10.3)$ & $3(7.69)$ & & \\
\hline \multirow{3}{*}{$\begin{array}{l}\text { NAC initiation } \\
\text { post-ingestion } \\
(\%)^{b}\end{array}$} & $<8$ hours & $31(64.6)$ & 19 (63.3) & $50(64.1)$ & 27 (69.2) & \multirow{2}{*}{0.777} & \multirow{2}{*}{0.513} \\
\hline & $>8$ hours & 15 (31.25) & $8(26.7)$ & $23(29.5)$ & $9(23.1)$ & & \\
\hline & Others $^{d}$ & $2(4.2)$ & $3(10)$ & $5(6.4)$ & $3(7.7)$ & NA & NA \\
\hline $\begin{array}{l}\text { Anaphylactoid } \\
\text { reactions }(\%)^{b}\end{array}$ & & $5(10.4)$ & $0(0)$ & $5(6.41)$ & $2(5.13)$ & 0.505 & 1 \\
\hline $\begin{array}{l}\text { Psychiatry review } \\
(\%)^{\text {b }}\end{array}$ & & 41 (85.4) & $29(96.7)$ & 70 (89.7) & $35(89.7)$ & 0.379 & 1 \\
\hline
\end{tabular}

\section{TABLE 1: Patient characteristics and results}

NC-not calculated; NAC - N-acetylcysteine; EDOU - emergency department observation units; g - grams

Statistical tests: ${ }^{\text {a }}$ 2-tailed Mann-Whitley U test; ${ }^{b}$ Fisher's exact test; ${ }^{c}$ Chi-Square test

d Others: include unknown time of ingestion and RSTI cases

We note that the majority of our patients ingested paracetamol for reasons of deliberate self-harm (81.3$100 \%$ ). For the comorbidities studied in our cohort, we found only three cases of known alcohol dependence in the "ward" group. About half of the patients in both groups took co-ingestants, with similar rates of common co-ingestants like alcohol, caffeine, and orphenadrine. There was no significant difference in the median paracetamol dose ingested or the time of NAC initiation post-ingestion.

Four patients had liver injury with peak AT > $150 \mathrm{IU} / \mathrm{L}$ (described in Table 2). Two of the patients (patients 1 and 2) had initial AT that is slightly elevated but $<2 x$ ULN. The other two patients developed hepatotoxicity (patients 3 and 4). All the patients recovered well. There were no cases of liver failure requiring a transplant and there were no fatalities.

\begin{tabular}{|c|c|c|c|c|c|c|c|}
\hline No. & Year & $\begin{array}{l}\text { ALT/AST (AT) (IU/L) } \\
\text { on presentation }\end{array}$ & $\begin{array}{l}\text { Highest } \\
\text { AT (IU/L) }\end{array}$ & $\begin{array}{l}\text { Paracetamol dose i } \\
\text { ingested }(\mathrm{g})\end{array}$ & $\begin{array}{l}\text { NAC initiation from } \\
\text { ingestion }<8 \text { hours }\end{array}$ & $\begin{array}{l}\text { NAC } \\
\text { duration } \\
\text { (hours) }\end{array}$ & $\begin{array}{l}\text { "Medical" } \\
\text { LOS (hours) }\end{array}$ \\
\hline 1 & $\begin{array}{l}2011 \\
\text { ward }\end{array}$ & $70 * / 38$ & 174 & 16 & Yes & 150 & 178 \\
\hline 2 & $\begin{array}{l}2014 \\
\text { ward }\end{array}$ & $58^{\star} / 60^{\star}$ & 469 & 20 & No & 45 & 129 \\
\hline 3 & $\begin{array}{l}2014 \\
\text { ward }\end{array}$ & $18 / 26$ & 1561 & 51.4 & Yes & 61 & 180 \\
\hline 4 & $\begin{array}{l}2013 \\
\text { EDOU }\end{array}$ & $9 / 23$ & 1894 & Unknown & Yes & 33 & 133 \\
\hline
\end{tabular}

\section{TABLE 2: Patients with acute liver injury and hepatotoxicity}

*Elevated ALT or AST;

EDOU - emergency department observation units; ALT - alanine aminotransferase; AST - aspartate aminotransferase; AT - aminotransferase; NAC - N-acetylcysteine; LOS - length of stay

Table 3 describes the outcome of the patients after admission to inpatient wards and EDOU. Five patients (12.8\%) were transferred from EDOU to the inpatient medical ward. Of these, two had extended NAC 
treatment, one for international normalized ratio (INR) of 1.4 without transaminitis and the other is described in table 2 (patient 4). The other three patients were admitted for (i) altered mental state secondary to orphenadrine, (ii) initial refusal for NAC in EDOU and (iii) right hypochondrial pain and raised INR without transaminitis respectively. "Medical" length of stay in the "ward" group was 55.2 hours, significantly longer by 31.9 hours compared to that in the EDOU group at 23.4 hours $(\mathrm{p}<0.0001)$. A subgroup analysis excluding patients with AT>150 IU/L did not show any difference for LOS (Table 3).

\begin{tabular}{|c|c|c|c|c|}
\hline & $\begin{array}{l}\text { Pre-EDOU inpatient } \\
\text { ward group }\end{array}$ & $\begin{array}{l}\text { Post-EDOU } \\
\text { inpatient ward } \\
\text { group }\end{array}$ & "Ward" group & EDOU group \\
\hline Cases & 48 & 30 & 78 & 39 \\
\hline Discharged & 42 & 23 & 65 & 23 \\
\hline Discharged against advice & 1 & 2 & 3 & 2 \\
\hline $\begin{array}{l}\text { Transferred to inpatient medical } \\
\text { ward }\end{array}$ & NA & NA & NA & 5 \\
\hline $\begin{array}{l}\text { Transferred to hospital psychiatric } \\
\text { facility }\end{array}$ & 4 & 2 & 6 & 3 \\
\hline $\begin{array}{l}\text { Transferred to external psychiatric } \\
\text { facility }\end{array}$ & 1 & 3 & 4 & 6 \\
\hline $\begin{array}{l}\text { "Medical" LOS (hours) median } \\
\text { (interquartile range) }^{\mathrm{a}}\end{array}$ & $55.2(42.2-61.6)$ & $54.5(39.5-63.0)$ & $55.2(39.6-62.6)$ & $23.4(22.4-24.0)$ \\
\hline $\begin{array}{l}\text { "Medical" LOS (hours.) median } \\
\text { (interquartile range); excluding liver } \\
\text { injury }(n=4)\end{array}$ & $\begin{array}{l}54.8(41.3-61.0) ;(n= \\
47)\end{array}$ & $\begin{array}{l}51.6(39.2-62.1) ;(n= \\
28)\end{array}$ & $\begin{array}{l}54.0(39.5-61.6) ;(n= \\
75)\end{array}$ & $\begin{array}{l}23.4(22.4-23.9),(n= \\
38)\end{array}$ \\
\hline
\end{tabular}

\section{TABLE 3: Patient discharge outcomes}

LOS - length of stay; EDOU - emergency department observation units

a from time of admission to discharge or transfer to a psychiatric service (in our hospital or external facility)

\section{Financial impact results}

Table 4 describes the average LOS (in days) and cost outcomes for the "ward" group and the EDOU group. We found a statistically significant savings of about $\$$ \$784 (S\$589-S\$966) between patients treated in the EDOU compared to patients treated in the inpatient wards.

\begin{tabular}{|l|l|l|}
\hline Outcomes & "Ward" group $(\mathbf{n}=78)$ & EDOU group $(\mathbf{n}=39)$ \\
\hline Average length of stay (days) & $3.2(2.3-4.4)$ & $2.0(1.2-3.4)$ \\
\hline Average cost (S\$) & $2,637(2,201-3,163)$ & $1,300(860-1,992)$ \\
\hline
\end{tabular}

\section{TABLE 4: Average actual length of stay and cost outcomes}

a Based on the actual length of stay in days (inclusive of stay in our hospital psychiatric facility)

S\$ - Singapore dollars

Table 5 details the average costs stratified according to outcomes in both "ward" group and EDOU group respectively. Of note, when a patient is transferred to an external psychiatric facility, the cost of stay in the external psychiatric facility is not computed. Patients who required hospital stay under our hospital psychiatric facility tended to incur increased costs. 


\begin{tabular}{|c|c|c|c|c|c|}
\hline \multicolumn{6}{|l|}{ Average cost by outcomes } \\
\hline All inpatient ward group $(n=78)$ & & Median (S\$) & Mean (S\$) & Smallest bill (S\$) & Largest bill (S\$) \\
\hline Discharged & 65 & 1,843 & 2124 & 745 & 5,644 \\
\hline Discharged against advice & 3 & 2,805 & 3023 & 2,441 & 3,823 \\
\hline Transferred to hospital psychiatric facility & 6 & 6,230 & 7876 & 2,314 & 17,474 \\
\hline Transferred to external psychiatry facility & 4 & 2,745 & 2837 & 2,543 & 3,314 \\
\hline EDOU group $(n=39)$ & & Median (S\$) & Mean (S\$) & Smallest bill (S\$) & Largest bill (S\$) \\
\hline Discharged & 23 & 707 & 730 & 551 & 986 \\
\hline Discharged against advice & 2 & NA & 838 & 719 & 956 \\
\hline Transferred to inpatient medical ward & 5 & 1,765 & 2,114 & 878 & 3,772 \\
\hline Transferred to hospital psychiatry facility & 3 & 2,696 & 5,575 & 1,835 & 12,196 \\
\hline Transferred to external psychiatry facility & 6 & 673 & 824 & 642 & 1,334 \\
\hline
\end{tabular}

TABLE 5: Average cost by outcomes for all inpatient ward and EDOU group patients

S\$ - Singapore dollars

\section{Discussion}

We found a "medical" LOS reduction of 31.9 hours or $57.8 \%$ and cost savings ( $\$ \$ 784$ per patient) without increased adverse effects when eligible patient groups are admitted to the EDOU instead of the inpatient wards.

To ensure that the "ward" and EDOU groups were comparable, we selected patients who required at least 21 hours of NAC and were suitable for admission to the EDOU that were instead admitted to the inpatient ward. We included patients from 2011 as historical controls to detect any differences in admitted inpatients characteristics prior to the start of EDOU from 2012. We did not find significant differences between the various groups. This assures us that the "ward" and EDOU groups were comparable for patient outcomes and allowed valid cost comparison.

Compared to Tang's study [7], the number of paracetamol-poisoned patients admitted to the hospital (ward and EDOU) was similar (77 and 76 per year respectively). The proportion of patients admitted to the EDOU showed a positive trend from $49 \%$ in 2013 to $75 \%$ in 2014 (70\%). In both EDOU cohorts, the patients were young (median age $<25$ ), mostly female (more than $75 \%$ ), had a median dose of $10 \mathrm{~g}$ and had low rates of liver injury and hepatotoxicity. We also note that the majority of patients who ingested paracetamol did so for deliberate self-harm (81.3-100\%), which is similar to the rates reported by another tertiary hospital [6]. This may be because our study population consisted mainly of those with single large ingestions (>95\%).

Although our study cohort included patients where NAC was initiated more than eight hours after acute ingestion, those with large ingestions (dose $>30 \mathrm{~g}$ ), as well as those with AT mildly elevated but $<2 \mathrm{x}$ ULN at presentation, they did not affect the final results. These patients had an apparently higher risk of developing hepatotoxicity but they were distributed amongst both groups [16]. There were only four patients who developed liver injury and excluding them from the comparison did not affect the LOS estimate (Table 2 and 3).

The "medical" LOS of patients in the "ward" group was 55.2 hours compared to the EDOU group at 23.3 hours, a significant finding on the background of rising healthcare costs and hospital bed shortage. A patient could save 31.9 hours ( $57.8 \%$ reduction) of admission if admitted to the EDOU instead of the ward. Tang et al. found a $65 \%$ reduction in LOS with an observation ward median LOS of 23 hours [7]. Beauchamp found LOS reduction of 27 hours for patients eligible for the observation ward but were instead admitted to the inpatient ward [10]. Offerman found that the LOS for patients with NAC was 67 hours [17], which is comparable to our patients with ward admission at 55.2 hours. The EDOU patient has the necessary interventions expedited due to the streamlining of medical care, psychosocial evaluation within the 23-hour stay.

Despite the limitations of financial data abstraction where we were not able to quantify the cost incurred from inpatient hospital psychiatric facility stay separately, we found that EDOU admission resulted in a statistically significant savings of $\$ \$ 784$ per patient. EDOUs have shown to result in LOS reduction and significant cost savings compared to inpatient care for a variety of medical conditions $[9,18]$. Poisoning related studies have also shown cost savings when there was consultation with a toxicology service or poison center for poisoning. Isoardi et al. described a reduction in 1350 bed days and $\$ 2.25$ million dollars over a 
period of two years at a tertiary hospital in Australia, following the establishment of a dedicated clinical toxicology unit [19]. Friedman et al. found that assistance by the poison center in Illinois reduced LOS by 0.6 days on average and potential savings of $\$ 2078$ dollars per 10 patients [20]. Although the cost savings and LOS shortening in our study cannot be generalized to all poisonings, it shows that cost savings and reduced LOS can be achieved when patients are admitted to an EDOU with support from a toxicology service. Using our study cohort, we have an average of 39 patients a year (117 for three years). From this average, we extrapolate an annual savings of $\$ \$ 30576$ for our hospital, from a savings of $\$ \$ 784$ per patient.

The retrospective nature of this study exposes it to the biases of missing data and inaccurate coding. Our sample size is relatively small which limits the strength of our study results. Bias may have been introduced as one investigator was used to extract the data. Factors influencing the decision for EDOU or ward admission may not have been clearly documented, concealing possible confounders. We did not follow-up patient outcome after discharge although a previous study noted that delayed adverse poisoning effects were negligible [11]. Lastly, our single-centre study limits the generalization of our results to other centres that do not have a similar set up.

\section{Conclusions}

Our study suggests that selected patients with paracetamol poisoning that require a standard course of NAC can be safely and effectively managed in an EDOU supported by a toxicology service. There is cost savings and decreased LOS without an increase in adverse effects. Further studies are needed to verify our results.

\section{Additional Information}

\section{Disclosures}

Human subjects: Consent was obtained by all participants in this study. Singapore Health Services Institutional Review Board issued approval 2017/2157. The SingHealth CIRB C has determined that the application as titled above qualifies for exemption according to its policies because of the following reason(s): 1. Your declaration that the research involves analysis of data available publicly/ dataset/ biological samples without identifiers. The SingHealth CIRB reference number for this study is 2017/2157. The documents reviewed are: a) CIRB Exemption Application Form dated 13 Mar 2017 The SingHealth CIRB has approved your request for waiver of informed consent based on ethical consideration. Please ensure that this study complies with your Institution policies. 1. Please note that an exempted study should be conducted in compliance with all applicable institutional policies, regulations and guidelines. Where necessary, approvals from the relevant authorities within your institutions should be sought before the conduct of the study. 2. Changes to the protocol that may affect the exempt status should not be initiated without prior approval by the SingHealth CIRB, except where necessary to eliminate apparent immediate hazard(s) to the study participants. Animal subjects: All authors have confirmed that this study did not involve animal subjects or tissue. Conflicts of interest: In compliance with the ICMJE uniform disclosure form, all authors declare the following: Payment/services info: All authors have declared that no financial support was received from any organization for the submitted work. Financial relationships: All authors have declared that they have no financial relationships at present or within the previous three years with any organizations that might have an interest in the submitted work. Other relationships: All authors have declared that there are no other relationships or activities that could appear to have influenced the submitted work.

\section{Acknowledgements}

The authors would like to thank Lim Mei Nai and Rachel Tan from the Finance department of Changi General Hospital for their help in drawing out the pricelist for 2018.

\section{References}

1. Mowry JB, Spyker DA, Brooks DE, Zimmerman A, Schauben JL: Annual report of the American Association of Poison Control Centers' National Poison Data System (NPDS): 33rd annual report. Clin Toxicol. 2015, 54:924-1109. 10.1080/15563650.2016.1245421

2. Altyar A, Kordi L, Skrepnek G: Clinical and economic characteristics of emergency department visits due to acetaminophen toxicity in the USA. BMJ Open. 2015, 5:e007368:Accessed: 13 July 2018: 10.1136/bmjopen2014-007368

3. Ponampalam R, Tan HH, Ng KC, Lee WY, Tan SC: Demographics of toxic exposures presenting to three public hospital emergency departments in Singapore 2001-2003. Int J Emerg Med. 2009, 2:25-31. 10.1007/s12245-008-0080-9

4. Chiu LQ, Lim BL, Vasu A, Phua DH, Goh HK: Poison exposure in the emergency department: a Singaporean experience. Hong Kong J Emerg Me. 2011, 18:197-203. 10.1177/102490791101800402

5. Arciaga GJ, Tan HH, Kuan KK, Mong RP, Kant A: A 24/7 hospital toxicology service: experience of a new start-up. Proceedings of Singapore Healthcare. 2018, 27:223-228. 10.1177/2010105818758783

6. Tan CJ, Sklar GE: Characterisation and outcomes of adult patients with paracetamol overdose presenting to a tertiary hospital in Singapore. Singapore Med J. 2017, 58:695-702. 10.11622/smedj.2016170

7. Tang D, Chan WL, Phua DH: Performance of an emergency department observation unit protocol in reducing length of stay for acetaminophen overdose: a retrospective study. Int. J Emerg Med. 2018, 11:48. 10.1186/s12245-018-0210-y

8. Judge BS, Ouellette LM, VandenBerg M, Riley BD, Wax PM: Utilization of observation units for the care of poisoned patients: trends from the Toxicology Investigators Consortium Case Registry. J Med Toxicol. 2016, 12:111-120. 10.1007/s13181-015-0498-4

9. Ross MA, Hockenberry JM, Mutter R, Barrett M, Wheatley M, Pitts SR: Protocol-driven emergency 


\section{Cureus}

department observation units offer savings, shorter stays and reduced admissions. Health Aff. 2013, 32:2149-2156. 10.1377/hlthaff.2013.0662

10. Beauchamp GA, Hart KW, Lindsell CJ, et al.: Performance of a multi-disciplinary emergency department observation protocol for acetaminophen overdose. J Med Toxicol. 2013, 9:235-241. 10.1007/s13181-0130310-2

11. Mong R, Arciaga GJ, Tan HH: Use of a 23-hour emergency department observation unit for the management of patients with toxic exposures. Emerg Med J. 2017, 34:755-760. 10.1136/emermed-2016-206531

12. Ministry of Health, Singapore. Admissions and outpatient attendances . (2018). Accessed: 13 July 2018: http://www.moh.gov.sg/content/moh web/home/statistics/Health Facts_Singapore/Admissions and Outpatient_Attendances.

13. Wolf SJ, Heard K, Sloan EP, Jagoda AS: Clinical policy: critical issues in the management of patients presenting to the emergency department with acetaminophen overdose. Ann Emerg Med. 2007, 50:292-313. 10.1016/i.annemergmed.2007.06.014

14. Musana KA, Yale SH, Abdulkarim AS: Tests of liver injury. Clin Med Res. 2004, 2:129-131. 10.3121/cmr.2.2.129

15. Persson HE, Sjöberg GK, Haines JA, de Garbino JP: Poisoning severity score. Grading of acute poisoning . J Toxicol Clin Toxicol. 1998, 36:205-213. 10.3109/15563659809028940

16. Wong A, Graudins A: Risk prediction of hepatotoxicity in paracetamol poisoning . Clin Toxicol. 2017, 55:879-892. 10.1080/15563650.2017.1317349

17. Offerman $\mathrm{S}$ : The clinical management of acetaminophen poisoning in a community hospital system: factors associated with hospital length of stay. J Med Toxicol. 2011, 7:4-11. 10.1007/s13181-010-0115-5

18. Abbass IM, Krause TM, Virani SS, Swint JM, Chan WY, Franzini L: Revisiting the economic efficiencies of observation units. Managed Care Magazine. 2015,

March:https://www.managedcaremag.com/archives/2015/3/revisiting-economic-efficiencies-observationunits.

19. Isoardi KZ, Armitage MC, Harris K, Page CB: Establishing a dedicated toxicology unit reduces length of stay of poisoned patients and saves hospital bed days. Emerg Med Australas. 2017 Jun, 29:310-314. 10.1111/1742-6723.12755

20. Friedman LS, Krajewski A, Vannoy E, Allegretti A, Wahl M: The association between U.S. Poison Center assistance and length of stay and hospital charges. Clin Toxicol. 2014, 52:198-206. $10.3109 / 15563650.2014 .892125$ 